vol. $19-n^{\circ} 2$ | 2003

Les initiatives de l'étranger et les nouveaux

cosmopolitismes

\title{
Peurs européennes, peurs malthusiennes
}

Detelina Tocheva

\section{(2) OpenEdition}

Journals

Édition électronique

URL : https://journals.openedition.org/remi/2946

DOI : $10.4000 /$ remi.2946

ISSN : $1777-5418$

Éditeur

Université de Poitiers

Édition imprimée

Date de publication : 1 juillet 2003

Pagination : $141-156$

ISBN : 2-911627-34-2

ISSN : 0765-0752

Référence électronique

Detelina Tocheva, "Peurs européennes, peurs malthusiennes », Revue européenne des migrations internationales [En ligne], vol. $19-\mathrm{n}^{\circ} 2$ | 2003, mis en ligne le 19 avril 2007, consulté le 15 avril 2022. URL : http://journals.openedition.org/remi/2946 ; DOI : https://doi.org/10.4000/remi.2946

Ce document a été généré automatiquement le 15 avril 2022.

(c) Université de Poitiers 


\title{
Peurs européennes, peurs malthusiennes
}

\author{
Detelina Tocheva
}

1 Le 14 mai 2002, dans une émission sur le futur élargissement de l'Union européenne aux pays de l'Est, la chaîne Arte diffuse des reportages sur quelques pays candidats à l'entrée dans l'Union. Une question y revient avec insistance : après l'adhésion, les habitants des pays de l'Est partiront-ils chercher du travail et s'installer chez leurs voisins occidentaux? Des scènes de la vie de Slovènes, de Polonais et de Roumains témoignent de l'existence de situations sociales très diverses. Lors du débat en direct qui suit, le commissaire européen à l'élargissement s'indigne du peu d'enthousiasme que mettent les États membres à «vendre l'élargissement " à leur population. La ministre polonaise des affaires étrangères explique, en direct de Varsovie, que tout porte à croire que les ressortissants polonais ne partiront pas dans les pays de l'Ouest européen pour y chercher du travail.

2 Pourquoi les pays membres sont-ils si réticents à «vendre l'élargissement » à leurs propres habitants? Pourquoi est-il primordial pour la ministre polonaise des affaires étrangères d'assurer l'Union européenne que ses compatriotes ne travailleront pas ailleurs qu'en Pologne? De quoi les travailleurs de l'Est sont-ils l'enjeu? Les réponses que ces questions appellent méritent d'être analysées.

3 Le débat sur l'ouverture du marché européen du travail aux nouveaux adhérents de l'Est n'est pas un sujet vedette dans le monde médiatique européen et, à la fois pour cause et par conséquent, il ne constitue pas un point de focalisation de l'opinion publique. La chaîne Arte représente une exception qu'expliquent ses affinités proeuropéennes ${ }^{1}$. Tant chez les États membres - à l'exception de l'Allemagne et de l'Autriche - que dans les pays candidats, la question est restée confinée au monde des experts, des instituts d'études de l'opinion, des analystes économiques, des spécialistes des migrations, des clubs de réflexion ${ }^{2}$. La situation est typique de tout un ensemble de domaines dans lesquels la légitimité de la supposée compétence de l'expertise l'emporte sur celle du supposé arbitraire du politique par des arguments d'impartialité et d'objectivité scientifiques. Et si, au bout du compte, la décision de ne pas octroyer, 
dans un premier temps, aux ressortissants des nouveaux pays adhérents le statut de travailleurs européens s'est avérée appartenir aux décideurs politiques, les experts se sont réservé la production des termes du débat.

4 Basé sur des enquêtes portant sur l'élargissement de l'Union européenne, plus approfondies dans le cadre de l'Estonie, de la Pologne et de la Hongrie, en particulier auprès des milieux politico-administratifs et des experts, l'article tente de mettre en lumière la construction de représentations et de catégories liées à cette question ${ }^{3}$. Ces dernières ressortissent à l'un ou à l'autre des deux grands registres, celui des normes construites comme objectives et celui des subjectivités. Le but de l'article n'est pas de soutenir ou d'invalider telle ou telle étude ou projection; il ne s'agit pas non plus de juger de leur impartialité et de leur scientificité. L'analyse du traitement de la question de la libre circulation des travailleurs de l'Est au sein de l'Union européenne vise à dresser un tableau de la structuration des représentations et des valeurs en jeu, ainsi que de rattacher cet exemple à un mode général d'appréhender des populations perçues comme différentes.

La norme européenne : une identité européenne minimale

5 La rhétorique de l'élargissement de l'Union européenne aux pays de l'Est, depuis le début des années 1990, s'est construite notamment sur la base d'un binôme simple: altérité/identité. La projection dans un ensemble imaginaire, en l'occurrence l'Europe élargie, implique pour les représentants de l'Union un autre à digérer, une différence à intégrer. Les objectifs de la préparation à l'adhésion, exprimés par des formules politico-techniciennes, telles que "démocratisation » et "institution building», sont aussi porteurs d'un message identitaire. La question sous-jacente pour l'Europe, derrière l'appareillage technocratique du processus, est de savoir comment faire pour que les pays de l'Est, considérés pendant un demi-siècle comme étant si différents de ceux de l'Ouest, se mettent à ressembler à ces derniers? L'adoption des normes européennes a alors été érigée en critère fondamental de la réduction de l'altérité, cette dernière globalement définie comme tenant de l'héritage communiste. L'adoption des normes communautaires s'inscrit dans deux registres en pratique d'importance égale : l'un du genre normatif, l'autre affectif (Tocheva, 2000). D'abord elle constitue un critère perçu comme objectif car identifiable, mesurable, quantifiable ; ce critère conforte la logique bureaucratique, notamment celle des institutions européennes, qui l'institue et selon laquelle les institutions se reproduisent. Ensuite le travail sur l'adoption des normes implique de nombreuses rencontres entre les représentants de l'Union et ceux des pays candidats, et a donc pour fonction intrinsèque leur rapprochement. Les États candidats ont déployé un effort politique, administratif et économique considérable afin d'adopter les règles européennes et d'acquérir la capacité de les appliquer; selon le glossaire européen, ils ont commencé la reprise de l'acquis communautaire ${ }^{4}$. Cet effort s'étend à une multitude de domaines relevant de la compétence de l'État : l'économie, les finances, l'agriculture, les transports, les statistiques, l'écologie... La norme étant construite comme la condition sine qua non de l'admission des pays de l'Est au sein de l'Union, c'est l'apprentissage de celle-ci par ceux-là qui est entré au centre des préoccupations européennes.

6 L'Union européenne, à travers sa Commission, a développé une pédagogie à l'intention des futurs adhérents de l'Est. Il n'avait pas été question d'une telle pédagogie lors des précédents élargissements. Ainsi, les opérations de «jumelage administratif $»^{5}$, qui sont l'incarnation même de cette pédagogie, visent à montrer aux fonctionnaires des pays 
candidats la manière dont les États membres mettent en œuvre les règles européennes, chaque pays selon ses modalités propres, et à leur suggérer les manières dont ils pourraient les appliquer chez eux. La dimension pédagogique de l'Europe a ainsi été pour la première fois explicitement formulée, une stratégie concrète élaborée, des outils spécifiques mis à sa disposition. La norme agissant comme une identité européenne minimale, tout fonctionne au moyen d'une reconnaissance par les candidats et les membres d'un besoin fondamental pour les Européens de l'Est d'apprendre à faire "à l'européenne " pour acquérir ainsi la légitimité d'entrer dans l'Union.

7 En 2001, dans les rapports annuels sur les progrès réalisés par chacun des pays de l'Est dans la reprise de l'acquis communautaire, la Commission européenne a constaté que pratiquement tous les pays avaient réalisé des progrès satisfaisants. Selon le critère de l'adoption des normes, rien ne tendait donc à remettre en cause leur future entrée dans l'Union, huit d'entre eux tablant sur une adhésion en 2004, les deux autres en $2007^{6}$. La même année, sous l'impulsion de l'Allemagne et de l'Autriche, les pays membres ont décidé de recourir à des " périodes de transition à la libre circulation des travailleurs " provenant des pays adhérents de l'Est ${ }^{7}$. La durée de cette période a été fixée à sept ans maximum à partir de leur entrée dans l'Union ${ }^{8}$. La période de transition signifie concrètement que durant sept ans, si l'État membre le décide, les ressortissants des nouveaux pays membres n'auront pas accès à son marché du travail, autrement dit ils ne seront pas considérés comme travailleurs européens, mais leur statut sera à convenir entre États sur le mode bilatéral.

8 Dans la rhétorique de l'élargissement, le registre de l'aplanissement des différences par l'adoption des normes s'ouvre en fait sur un autre, moins technique, moins mesurable, mais bien présent à l'esprit des responsables des États membres et des pays candidats, celui des peurs et des méfiances à l'égard du nouveau venu. Les fonctionnaires de la Commission européenne discutent volontiers du premier avec le chercheur, mais deviennent beaucoup moins volubiles dès qu'il est question du second; ce sont les "questions politiques", à comprendre les plus sensibles, les plus difficiles, celles qui divisent les États membres, les plus dérangeantes. Les travailleurs des pays candidats envahiront-ils les actuels pays membres de l'Union? est une de ces questions suscitant des appréhensions et des méfiances. Cette question apparaît autonome par rapport à celle de l'adoption et de la mise en œuvre des normes communautaires à l'Est. Elle semble se mouvoir selon sa logique propre.

Travailleur européen : une catégorie à sémantique incertaine

9 L'appartenance à l'Union implique théoriquement quatre libertés de circulation sur son territoire: celle des marchandises, celle des capitaux, celle des services et celle des personnes. La liberté de voyager dans les pays d'Europe de l'Ouest, d'y travailler ou de s'y installer était l'une des libertés les plus espérées par les populations de l'Est depuis la fin des régimes communistes. Accéder à cette liberté signifiait alors que la période de fermeture des pays sous contrôle soviétique était terminée. Cependant, il n'y a pas eu de rupture nette. Les pays de l'Union européenne ont, dans un premier temps, maintenu les visas pour tous les types de séjour pour les ressortissants des pays de l'Est, candidats à l'Union européenne. Progressivement les visas de court séjour ont été supprimés par la plupart des États membres ${ }^{9}$, qui ont tout de même retenu une exigence de moyens suffisants, en se réservant le droit de renvoyer dans leur pays d'origine les individus n'y répondant pas. Une sélection économique s'est donc 
imposée. La circulation des travailleurs est quant à elle soumise à une série de conditions qui sont de la compétence de chacun des États membres, sans que des règles européennes communes soient actuellement appliquées ${ }^{10}$.

La question de l'ouverture du marché du travail communautaire aux travailleurs des pays de l'Est a donné lieu, au sein des institutions européennes et dans les milieux politico-administratifs et d'experts des États membres et candidats, à un jeu de constructions de profils économico-psycho-sociologiques des populations, de typologies de leur mobilité potentielle. L'élaboration, délibérée ou non, d'une figure du travailleur de l'Est, s'appuie sur une vulgate d'études sociologiques, démographiques, économiques et sur une considérable masse d'enquêtes d'opinion effectuées à l'Est et à l'Ouest de l'Europe. Il est à noter qu'aux côtés des institutions européennes, du Bureau International du Travail et de l'OCDE, ce sont les organismes d'expertise et de recherche allemands et autrichiens qui se sont montrés les plus actifs en Europe occidentale, l'Allemagne et l'Autriche étant les pays frontaliers des candidats de l'Est ${ }^{11}$. Les exemples de mises en garde contre un afflux de travailleurs sont trop nombreux pour que l'on puisse les citer tous ici ; mais celle formulée par le BIT au moment même de la chute des régimes communistes est représentative du ton général de toutes celles du début des années 1990: "Il n'est pas exagéré de prévoir que la politique de "perestroika" menée en Union soviétique et dans les autres (anciens) pays socialistes européens pourrait provoquer un exode massif de travailleurs de ce pays à la recherche de possibilité d'emploi à l'étranger » (Okolski, 1991 : 35). Comme nous le verrons par la suite, la position générale de l'expertise à l'Ouest a évolué vers la fin des années 1990.

11 Le jeu d'échange d'arguments et de contre arguments entre représentants des pays membres de l'Union et des pays candidats - un jeu qui se matérialise à l'occasion des sommets politiques, des négociations "techniques", des parutions d'études et de sondages, d'articles dans la presse, et par tout autre moyen qui permet de les rendre publics, y compris l'entretien accordé à un chercheur, - repose sur un accord plus ou moins tacite entre les protagonistes des deux côtés. Les pays de l'Est s'y trouvent $a$ priori définis comme des pays d'émigration avérée ou potentielle, ceux de l'Ouest comme des pays d'immigration, les premiers étant moins riches que les seconds. Dans le contexte de leur future adhésion à l'UE, les ex-pays communistes s'attachent à contredire la thèse d'un exode pour neutraliser l'idée de leur dangerosité qu'induit ce type de prévisions, comme l'a fait la ministre polonaise des affaires étrangères sur Arte. Ainsi, aussi paradoxal que cela puisse paraître quand on connaît le principe de l'Union qui veut que l'espace européen soit mis en commun pour tous ses habitants, d'un côté la volonté de l'Union européenne d'empêcher l'accès des travailleurs de l'Est à l'emploi dans l'Union, d'un autre côté celle des représentants politico-administratifs de l'Est qui d'ailleurs ne souhaitent pas voir leur pays déserté par la population en âge de travailler, étant donné le vieillissement démographique et toutes les craintes que cela implique - s'accordent au fond sur un même message : moins la libre circulation des travailleurs entre les actuels et les futurs membres de l'Union sera appliquée, mieux ce sera pour tous. Autrement dit, pour eux, moins l'Europe fonctionne pour les travailleurs de l'Est, moins les uns et les autres courent de dangers. L'Union européenne se trouve en l'occurrence confortée par la négation de ses propres principes.

Les populations de l'est, des gens qui « restent chez eux » 
12 Dans les pays candidats, que ce soit à l'Institut polonais d'études du travail et de la sécurité sociale à Varsovie, au Centre national hongrois de recherche sur le travail à Budapest, à l'office national estonien des statistiques à Tallinn, au bureau d'un responsable administratif du ministère des affaires sociales ou dans celui du conseiller d'un ministre, la même conviction est mille fois réaffirmée : les travailleurs de notre pays ne bougeront pas après l'entrée dans l'Union. Des arguments de tout ordre se trouvent déployés, statistiques et sondages à l'appui. La Pologne, dont les travailleurs constituent aux yeux des dirigeants allemands l'une des principales menaces pour l'équilibre de leur pays ${ }^{12}$, ne dissimulerait en fait aucun danger. Selon un conseiller au ministère polonais des affaires sociales et du travail, toute cette peur serait le produit d'une mauvaise manière de formuler les questions dans les sondages, ce qui aurait induit les responsables allemands en erreur ${ }^{13}$. Des chercheurs de l'Institut polonais d'études du travail et de la sécurité sociale, un organisme public jouant le rôle de thinktank au service des politiques polonais, affirment dans une communication intitulée «La migration économique contemporaine de et vers la Pologne », avec de multiples statistiques et observations sociologiques à l'appui : "Nous croyons que la majorité du peuple polonais voudrait voir et voit son avenir en Pologne, même si les Polonais veulent visiter des pays étrangers" (Domaradzka et Rajkiewicz, 2000). En ce qui concerne la Hongrie, elle se vante d'afficher le meilleur niveau économique des pays de l'Est, et un enviable taux de chômage aux alentours de 7\%. Dans son rapport annuel pour 2000, le Centre national hongrois de recherche sur le travail souligne que malgré les avantages que procurent l'emploi et l'expérience acquise à l'étranger, ainsi que les salaires plus élevés qu'en Hongrie, moins de 1\% des travailleurs hongrois occupent un emploi à l'étranger ; ces travailleurs migrants étant peu nombreux ne mériteraient pas une très grande attention de la part de la politique de l'emploi (Laky, 2000 : 45-50). Les hauts fonctionnaires hongrois mettent en avant le fait que les migrations liées au travail sont limitées aux régions frontalières avec l'Autriche, et qu'elles sont quotidiennes, que les travailleurs ne s'y installent pas durablement. Leurs homologues estoniens soulignent que l'Estonie est un petit pays avec à peine 1,39 millions d'habitants qui ne comptent pas émigrer. " Il y a un marin estonien dans chaque port du monde ", écrivait Hemingway. "Aujourd'hui les Estoniens cherchent du travail en Estonie ", réplique une fonctionnaire elle-même en charge de préparer la reconnaissance mutuelle des diplômes entre l'Estonie et les pays de l'Union.

Les Européens de leur côté appellent à la prudence : depuis 1970, année où le solde migratoire pour l'ensemble des États membres était négatif $(-1,1 \%$ o), il a atteint un pic à $2,8 \%$ en 1990 et reste positif à 1,8 \%o en 2000. Pour les pays de l'Est en revanche, ce solde est pour certains égal à zéro, pour d'autres légèrement supérieur, (la Slovénie fait figure d'exception avec $1,4 \%$ pour 2000), mais il est le plus souvent négatif (Commission européenne, $2001 \mathrm{~d}: \mathrm{J}-5)$. « En raison de l'évolution économique générale et de la période de transition que connaissent les pays d'Europe centrale et de l'exUnion soviétique, les tendances de la migration dans les États membres de l'UE ont radicalement changé ces derniers temps. Dans les pays du Nord de l'UE, l'entrée et la présence de non-nationaux se sont accrues - mais surtout en raison des regroupements familiaux et de l'augmentation du nombre de migrants de la deuxième génération -, alors que les États membres du Sud sont devenus des pays d'immigration » (Commission européenne, 2001 b : 86). Les immigrés de l'Est sont non seulement présents en Europe de l'Ouest, mais arrivent même à inverser la situation dans les pays du Sud de l'Europe, traditionnellement terre d'émigration au $\mathrm{Xx}^{\mathrm{e}}$ siècle. La 
plupart des analyse ${ }^{14}$ précisent qu'il s'agit essentiellement de ressortissants de pays issus de l'ex-Yougoslavie, de la Turquie ou de l'ex-URSS, et que les dix pays candidats de l'Est ne constituent pas une redoutable source d'émigration. La thèse d'un déplacement massif à l'instar des flux d'immigration déjà connus en Europe occidentale se trouve, depuis le milieu des années 1990, globalement discréditée, tant par les candidats que par les membres de l'UE. De nombreux travaux réalisés notamment en Allemagne et en Autriche concluent également qu'une importante vague d'émigration vers l'UE serait improbable, mais que ces deux pays resteraient les plus concernés devant absorber $80 \%$ de l'ensemble des futurs travailleurs immigrés ${ }^{15}$. Néanmoins, le message prégnant en deçà et au-delà du texte "objectif » de l'expertise construit le ressortissant Esteuropéen en général comme un travailleur immigré potentiel après l'élargissement: poser la question comme problématique en est déjà une expression sans équivoque. Mais de quel type d'immigration s'agit-il ?

Les travailleurs espagnols, portugais et polonais : des catégories pour signifier à la fois l'identique et le différent

14 Un discours généralisé au sein de la haute administration des pays candidats tend à comparer ces derniers à ceux d'Europe du Sud en ce qui concerne les effets possibles de l'accès des travailleurs à l'emploi dans l'Union. Il s'agit de suggérer aux États membres que leurs craintes actuelles d'être envahis par des travailleurs de l'Est seraient du déjà vu et que, comme dans le cas de l'Espagne et du Portugal, elles seraient infondées, car l'exode tant redouté après leur entrée ne s'est pas produit ${ }^{16}$. Ce discours a la particularité d'évoquer un rapprochement entre la situation des futurs adhérents de l'Est et celle d'anciens candidats occidentaux. Mais un tel rapprochement n'est évoqué dans aucun autre domaine ressortissant à la future adhésion ; bien au contraire. Pour se distancer des États membres, les responsables politico-administratifs de l'Est insistent sur les énormes efforts à mettre en œuvre pour répondre aux critères européens, efforts qu'aucun des actuels pays membres, y compris l'Espagne et le Portugal, n'aurait eu à accomplir. Ils limitent la comparaison avec l'Espagne et le Portugal au seul domaine de la libre circulation des travailleurs; dans le système de représentations en œuvre notamment dans la haute administration polonaise, estonienne et hongroise, aller au-delà pourrait nuire à l'image de leur pays qui ne tient pas à être associé aux "mauvais élèves » de l'Europe que sont traditionnellement réputés être les pays du Sud. Le jeu d'association à une catégorie préexistante devient en même temps un jeu de dissociation par rapport au contenu négatif qu'elle comporte.

Du point de vue de l'Union également le parallèle avec les pays du Sud a ses limites, mais des arguments différents se trouvent mobilisés. « Aujourd'hui les flux migratoires concernent de nouveaux types de population (personnes hautement qualifiées, migration temporaire, étudiants). [...] l'exode massif vers l'Europe occidentale/ septentrionale après les événements du début des années 1990 ne s'est pas matérialisé [...] 》 (Commission européenne, $2001 \mathrm{~b}:$ : 86), contrairement à l'installation massive de Portugais et d'Espagnols dans les pays au Nord de la péninsule ibérique dans les années 1960 et 1970, à la suite de bouleversements politiques et économiques, avant même l'adhésion du Portugal et de l'Espagne à la Communauté européenne.

Il s'agirait donc aujourd'hui de flux plus maîtrisés et plus faibles, mais aussi de populations ayant des caractéristiques différentes de celles des immigrés espagnols et portugais de l'époque. Si ces derniers se sont majoritairement insérés au bas de l'échelle sociale, en occupant des emplois qui exigeaient une faible qualification, il n'en 
va pas de même des immigrés de l'Est. En France par exemple, l'immense majorité des travailleurs originaires des pays de l'Est qui ont reçu le statut de travailleurs permanents depuis 1990 sont classés dans la catégorie «cadres» (OMI, 2000). « Pour tous les pays candidats, les indicateurs montrent que le pourcentage de personnes ayant un niveau d'instruction correspondant au minimum au niveau du deuxième cycle de l'enseignement secondaire est plus élevé que pour l'UE-15 dans son ensemble $(63,5 \%) »(\text { Commission européenne, } 2001 \text { a: 402-403) })^{17}$. La différence est donc statistiquement parlant qualitative et elle est perçue comme telle par les responsables politiques des États membres ${ }^{18}$.

En cherchant des parallèles possibles dans l'histoire française, nous pouvons nous interroger : dans quelle mesure l'expérience de l'immigration polonaise en France dans les années 1920-1930 serait éclairante dans les nouvelles conditions. Une enquête menée en 1951-1952 par l'INED sur l'adaptation des immigrés polonais et italiens en France s'attachait à saisir le degré d'intégration de ces populations d'immigrés, se basant sur des « signes d'assimilation » - la maitrise de la langue, les relations avec les voisins français, la " participation à la vie sociale ", la tenue vestimentaire, la cuisine, la pratique de la religion et du «folklore» d'origine, etc. (Girard et Stoetzel, 1953). Dans sa préface Alfred Sauvy affirmait, avec une dose typique de bon sens politique, que « les dualités persistantes [entre la population française et immigrée] sont considérées comme des causes de tension, qu'il convient de faire cesser » (Girard et Stoetzel, 1953 : XIII). L'étude concluait que l'assimilation - terme consacré à l'époque - des Italiens ouvriers du bâtiment, commerçants et agriculteurs, ainsi que celle des Polonais agriculteurs et mineurs de fond dans les Houillères du Nord et du Pas-de-Calais, était en bonne voie. Les immigrés polonais, arrivés en masse après la Première Guerre mondiale, avaient des origines modestes. Dans les 129 familles d'agriculteurs étudiées dans l'Aisne, parmi les chefs de famille " un seul a dépassé dans sa jeunesse le niveau des études primaires : il s'agit d'un ancien officier qui a monté une petite exploitation avicole » (Girard et Stoetzel, 1953 : 381). Quant aux mineurs dans le bassin houiller du Nord et du Pas-de-Calais, on apprend qu' « à titre exceptionnel seulement, ils ont fait des études dépassant le niveau du cycle primaire ", les pères de ces mineurs ayant été pour moitié des mineurs eux-mêmes, pour moitié de petits cultivateurs (Girard et Stoetzel, $1953: 450$ ). Ces immigrés des années 1920 et 1930 s'intégraient donc plutôt facilement, le décalage culturel n'étant pas assez significatif pour perturber leur installation au sein de la société française. Ils avaient pour autre caractéristique d'occuper le plus bas échelon socioprofessionnel dans la société d'accueil. Cette expérience du début du siècle est donc à rapprocher de celle de l'immigration portugaise et espagnole évoquée par les pays candidats, tant sur le plan de la proximité culturelle - une intégration facile - que sur celui de l'intégration socioprofessionnelle - la première et souvent la deuxième génération n'ont eu pratiquement aucune chance d'appartenir aux élites.

En Europe, l'opposition Nord-Sud tend à se résorber et opère sa transmutation en une opposition Ouest-Est ; cette dernière ne correspond plus à celle de la guerre froide. La nouvelle configuration est la transcription en termes topographiques d'une taxinomie sous-jacente des populations européennes, qui ne recouvre pas la signification de l'opposition Nord-Sud. Le stéréotype de l'immigré de l'Est qui est en train de se construire dans le contexte du futur élargissement, en partie par la magie des chiffres, en partie sous l'effet d'images semi-littéraires, semi-journalistiques, en partie par des expériences concrètes, n'est pas la réplique actualisée de celui du gardien d'immeuble 
ou de l'ouvrier du bâtiment portugais ou espagnol, ni celle du mineur polonais. Si jusqu'à présent l'Europe des quinze a accueilli une immigration de l'Est, souvent illégale, occupant des emplois subalternes et "au noir", ce n'est pas ce type de population qui pose problème aux États membres au bout du compte. En fait, l'Union n'a pas de précédent lui permettant de juger des résultats de l'ouverture de son marché du travail aux nouveaux adhérents de l'Est. Le profil type du travailleur Est-européen issu des pays futurs adhérents à l'Union, tel qu'il se forme dans les représentations des responsables Ouest-européens, diverge de celui de l'immigré traditionnel. Il est en ceci inquiétant qu'il induit un rapport de domination avec les populations Ouesteuropéennes, un rapport dont celles-ci n'ont jamais fait l'expérience, étant généralement habituées à une immigration dont la première génération restait dans les basses strates de la société d'accueil. Le hors-texte des expertises qui parle aux dirigeants politiques situe la dangerosité de l'immigré potentiel de l'Est dans sa capacité à s'intégrer au sein des classes moyennes et des élites du pays d'accueil. L'enjeu de l'ouverture du marché du travail européen aux ressortissants de l'Est réside, plus que dans la sauvegarde de l'équilibre des régions frontalières, dans la maîtrise de ce rapport de domination.

Le social et le national

19 L'élargissement à l'Est, et notamment la question des travailleurs, est pensé par les membres de l'Union et par les candidats essentiellement sur le mode national. Quelques phénomènes sont symptomatiques de ce caractère qui n'autorise, dans la pratique, à considérer la dynamique de l'élargissement qu'en tant que produit des positions d'États nationaux. En premier lieu, l'adoption de périodes de non-application de la libre circulation aux travailleurs de l'Est s'est effectuée sous l'insistance de l'Allemagne et de l'Autriche. Les représentants des pays candidats perçoivent cette décision non pas comme communautaire et commune, mais comme insufflée par les dirigeants des deux États membres qui constituent la frontière orientale de l'Union, et qui se sentent par conséquent les plus menacés par un afflux de travailleurs. Ensuite, durant la période de transition, l'accession des travailleurs de chaque nouveau membre de l'Est à l'emploi dans l'Union européenne sera traitée par chaque actuel État membre selon le modèle bilatéral. La Grande-Bretagne, l'Allemagne, les Pays-Bas et d'autres se montrent déjà " ouverts » à certains types de travailleurs de l'Est : des spécialistes de l'informatique et des nouvelles technologies de l'information par exemple; il s'agit donc d'un système de quotas selon les professions que chaque État membre gère à sa guise.

En troisième lieu, mais tout aussi parlant, est le fait que chaque pays candidat, à partir de son exemple national, essaie de prouver qu'en tant que nouvel adhérent, il ne sera pas une catastrophe pour l'emploi dans l'Union européenne, ni une source de «dumping social». Par ailleurs, chaque pays candidat tient à négocier seul son adhésion, à être évalué individuellement et ne s'envisage pas en tant que partie d'un ensemble, alors que l'Union a mis en place une "stratégie de pré-adhésion " à l'intention de tous les candidats. Le refus de l'homogénéisation est particulièrement manifeste à travers la question des travailleurs. Les représentants estoniens par exemple ne manquent pas de se singulariser par rapport à la Pologne, une menace aux yeux des dirigeants allemands, en arguant que leurs voisins nordiques, membres de l'Union, seraient prêts à accueillir les travailleurs estoniens à bras ouverts; mais que de toute manière ceux-ci ne seraient pas nombreux. Cette affirmation que l'on entend souvent dans la haute administration estonienne est révélatrice du système de représentations et de la structuration des valeurs, partagés par l'Est et l'Ouest. Les pays 
candidats s'y trouvent classés selon leur degré de dangerosité : la faiblesse numérique de la population et son immobilité deviennent des valeurs positives, permettant de repérer le pays le plus inoffensif. Les pays membres s'y voient ordonnés selon leur degré d'ouverture : les pays scandinaves et la Grande-Bretagne seraient plus " ouverts » que l'Allemagne et l'Autriche, la méfiance et la fermeture étant construites en valeurs négatives, mais vues comme compréhensibles et inhérentes à l'élargissement. Ces représentations et ces valeurs informent un modèle de traitement de la question sociale que pose l'élargissement immuablement dans un rapport entre États nationaux, l'Union européenne n'y étant pas une entité aussi univoque et dense que les États qui la composent.

21 L'incapacité de penser en commun le social, et donc la seule existence d'un mode national pour son traitement, s'est en effet montrée, jusqu'à présent, constitutive du fonctionnement de l'Union. L'élargissement à l'Est constitue une sorte de catalyseur, permettant de constater qu'en Europe le social relève du domaine exclusif des États. L'inexistence d'une protection sociale commune, d'ailleurs interprétée par les pays candidats comme le thème tabou de l'Union, témoigne également de ce monopole national du domaine social. La coordination existant aujourd'hui entre les caisses nationales de sécurité sociale des États membres - qui s'opposerait dans un système unique à leur mise en commun - est symptomatique du principe selon lequel chaque État est maître chez lui pour gérer son domaine social. Le social n'est pas communautaire, il est inter-étatique ; social se confond donc avec national. Si la notion de solidarité nationale sur le plan social fait référence à la répartition des richesses allant généralement dans le sens riches-pauvres, une telle "solidarité européenne " n'est pas envisageable, aucun pays membre ne souhaitant payer pour les habitants moins riches d'un autre. Ce principe, selon lequel le social appartient au national, est devenu pour ceux des représentants des pays candidats qui sont amenés à connaître le fonctionnement de l'Union, un schème pour penser le social en Europe ; ils l'admettent comme un principe tacite mais fondamental. Dans ce domaine notamment, le politique n'aurait pas vécu sa "mutation européenne $»^{19}$. C'est au regard de cette prééminence du social en tant que strictement national que la notion d'union européenne prend une signification particulière à l'Est.

Conclusion

Une fois l'adhésion des pays de l'Est réalisée, tout porte à valoriser leur population en tant que consommateurs et producteurs : union économique et douanière, normes communes appliquées aux marchandises et aux services, possibilité de circuler en tant que touriste dans l'espace européen muni uniquement de son passeport... La figure de l'Européen de l'Est en tant que travailleur à l'Ouest paraît en revanche incompatible avec ces rôles positifs. Le travailleur venu de l'Est, en moyenne plus pauvre que celui de l'Ouest, est construit comme un homme potentiellement néfaste; une période d'adaptation (ou de «transition») lui est indispensable pour qu'il devienne acceptable. Le raisonnement relève du truisme : le travail étant devenu une marchandise rare dans l'Union européenne, un travailleur plus pauvre et mieux éduqué que la moyenne, ayant les mêmes droits, est susceptible de provoquer du «dumping social » et d'aggraver le taux de chômage; il est donc un homme indésirable car il menacerait le bien-être économique et social du pays (ou de l'Union) qui l'accueillerait. Il risquerait de rendre l'emploi encore plus déficitaire, sans que le système en place puisse résolument remédier à ce déficit. Les études évoquées supra affirment également que dans quelques années seulement l'Europe éprouvera un grand besoin de main-d'œuvre qualifiée. 
Cependant les prévisions des experts ne rencontrent dans les faits que la précaution des politiques ; la gestion du présent demeure imprégnée par la méfiance.

En 1798, dans son Essai sur le principe de population, Malthus exprimait la conviction que "l'inégalité naturelle entre le pouvoir de multiplication de la population et le pouvoir de production de la terre » faisait obstacle à la " perfectibilité de la société » (Malthus, 1980 [1798] : 26). Le bonheur d'un pays dépendrait du rapport entre l'accroissement des moyens de subsistance et l'accroissement de la population. Un parallèle avec notre problème semble pertinent. Procédant de l'idée qu'en Europe la capacité de créer de l'emploi (celle-ci se substitue au pouvoir de production de la terre dans le modèle malthusien) est limitée, l'acte d'empêcher, dans un premier temps, les futurs adhérents de l'Est d'accéder à l'emploi dans l'Union relève d'une sorte de gestion préventive du risque qu'est supposée comporter une population pour une autre.

Si l'adoption des normes juridiques est une technologie visant l'aplanissement des différences de l'autre afin de le rendre compatible - et il est indispensable de préciser ici que la logique européenne, telle qu'elle se manifeste dans le fonctionnement de l'Union, consiste à rendre, par la négociation, les différences compatibles - sa mise à distance par rapport au marché de l'emploi a pour objectif la neutralisation du danger social qu'il est supposé constituer.

Lorsque l'altérité procède d'une différence dans le niveau social, l'autre est perçu comme une menace pour l'équilibre, pour l'ordre social et pour le bien-être, car il pourrait s'accaparer un bien déficitaire - le travail. Le fait qu'il s'agit, dans le cas de l'élargissement de l'Union, d'une menace supposée neutralisable à terme (après une " période de transition » conduisant à une amélioration économique) n'entrave en rien la validité du paradigme. Les décideurs de l'Union européenne pratiquent, à l'égard des pays candidats, une sorte de contrôle des dangers potentiels. Bien plus que la marchandise hors norme, c'est l'être humain potentiellement dangereux qui est en cause. Alors, à l'instar de Malthus, l'Europe cherche une solution rationnelle à sa peur de manquer. Cette solution rendue par le signifiant "période de transition » a pour signifié la normalisation sociale de l'homme. Sa première étape est conçue comme une tenue à l'écart, la seconde comme une intégration, une fois son handicap supposé corrigé. Ce mode presque clinique de programmer l'intégration de nouvelles populations dans l'Union européenne apparait comme étant la réponse à la question du dépassement de l'altérité sociale, et donc de la méfiance et de la peur qu'elle engendre. Dans le cas de l'élargissement européen l'anormalité sociale de l'autre se voit traitée par l'exclusion de la possibilité pour les ressortissants de l'Est d'endosser le rôle de travailleurs européens. L'anormalité sociale du travailleur de l'Est a ceci d'original qu'elle n'est pas pensée comme rédhibitoire à sa participation à part égale dans des domaines autres que l'emploi ; il y a donc reconnaissance d'une normalité par ailleurs. L'isolement est sélectif; il est mis en œuvre là où la mesure exacte ne reçoit que peu de crédit, où elle paraît même incongrue, et où la méfiance devient la règle. Voilà comment la norme estimée objective est invalidée pour céder la place aux peurs européennes qui, à deux siècles de distance, sont si proches des peurs malthusiennes. 


\section{BIBLIOGRAPHIE}

COMMISSION EUROPÉENNE (2001 a) Annuaire Eurostat. Données 1990-2000.

COMMISSION EUROPÉENNE (2001 b) Annuaire Eurostat. Le guide statistique de l'Europe. Données 1989-1999.

COMMISSION EUROPÉENNE (2001 c) Free movement of workers in the context of enlargement, Note d'information, 6 mars 2001.

COMMISSION EUROPÉENNE (2001 d) Eurostat. Statistiques sociales européennes. Démographie 2001.

DOMARADZKA Ewa, RAJKIEWICZ Antoni (2000) Contemporary economic migration from and to Poland, IPISS, Varsovie (communication).

DUPÂQUIER Jacques, LAULAN Yves-Marie (2000) La population européenne et ses problèmes, Revue de l'Institut de Géopolitique des Populations, $\mathrm{n}^{\circ} 1$.

FERRY Jean-Marc, THIBAUD Paul (1992) Discussion sur l'Europe, Calmann-Lévy.

GIRARD Alain, STOETZEL Jean (1953) Français et immigrés. L'attitude française. L'adaptation des Italiens et des Polonais, INED, PUF.

LAKY Terèz (2000) Labour market report, 2000. Main trends in labour market demand and supply, National Centre for Labour Research and Methodology, Budapest.

MALTHUS Thomas Robert (1980 [1798, Londres]) Essai sur le principe de population, introduction par Eric Vilquin, INED.

OCDE (2003) International Mobility for the Highly Skilled, OCDE, Paris.

OKOLSKI Marek (1991) La nouvelle donne migratoire en Europe de l'Est, Revue Européenne des Migrations Internationales, vol. 7, n², 1991.

OMI (2000) OMISTATS. Annuaire des migrations 2000.

TOCHEVA Detelina (2000) Élargissement de l'Union européenne et mutation de l'État bulgare. Approche anthropologique d'une opération de jumelage administratif entre les ministères de l'agriculture bulgare, français et britannique, mémoire de DEA à l'EHESS de Paris, mémoire non publié (directeur Marc Abélès, tutrice Irène Bellier).

\section{NOTES}

1. En France, le journal Le Monde notamment a publié, depuis 2002, une dizaine d'articles où la position exprimée était globalement proche de celle des instituts d'étude et des divers groupes de réflexion traditionnels des pays membres de l'Union. L'article de C. Rallot «La déferlante migratoire n'aura pas lieu » (Le Monde Économie, le 10 déc. 2002) et celui de N. Bourcier « Ce que l'élargissement va changer en France » (le 24 oct. 2002) en constituent les meilleurs exemples.

2. En France, l'Institut de Géopolitique des Populations, une sorte de club de réflexion intégrant des universitaires et des personnalités politiques, a organisé un colloque le 10 décembre 1999, où l'avis partagé par les participants fut que les populations d'Europe de l'Est ne menaçaient pas l'Ouest par une émigration massive. Voir Jacques Dupâquier, 
Yves-Marie Laulan, (2000). Voir en particulier les contributions de Gérard-François Dumont (pp. 17-42) et de Pierre-Jean Thumerelle (pp. 91-106).

3. Cet article s'inspire, entre autres, d'une recherche de terrain réalisée de mars à novembre 2001 pour la Mission Recherche (DREES) du Ministère de l'Emploi et de la Solidarité (convention 03/01). Elle a notamment donné lieu à l'élaboration d'un rapport intitulé « Les enjeux sociaux et de protection sociale dans le processus d'élargissement européen. Le cas de trois pays candidats : Pologne, Hongrie, Estonie ", suivi d'un état de la recherche sur les enjeux sociaux et de protection sociale en Pologne, en Hongrie et en Estonie. Directeur scientifique de l'étude : Jean-François Gossiaux (LAIOS, EHESS). 4. L'acquis communautaire se constitue de 30000 pages de directives, de règlements et de traités, et de 70000 pages de « soft law », comprenant les accords d'association et la jurisprudence de la Cour européenne de justice.

5. Mises en œuvre depuis 1998.

6. La primauté du premier sur le second groupe quant à la date de l'adhésion est souvent rendue, au sein des institutions européennes, par la terminologie des « vagues ». La « première vague » comprend huit pays : Pologne, Hongrie, République tchèque, Slovaquie, Slovénie, Lituanie, Lettonie et Estonie. La " deuxième vague " réunit la Bulgarie et la Roumanie. Aux yeux des représentants bulgares et roumains, ce classement en « vagues » n'est pas sans contenir une certaine dose de péjoration à l'égard de ceux qui se trouvent relégués dans la « deuxième vague ».

7. L'Union européenne comprend 250 millions de personnes en âge de travailler, les dix pays candidats de l'Est en ont en commun 70 millions. Source : Commission européenne, Free movement of workers in the context of enlargement, Note d'information, 6 mars 2001.

8. Pendant deux ans, à compter de l'adhésion d'un pays, les actuels États membres pourront appliquer des exigences nationales à l'égard des travailleurs de chaque nouvel État membre. À l'issue de ces deux ans chaque actuel État membre réexaminera la situation de son marché du travail et aura la possibilité de reconduire les limitations à la libre circulation des travailleurs du nouvel État membre jusqu'à cinq ans, à compter de sa date d'adhésion. Enfin, au bout de ces cinq ans, ceux des États membres, qui estimeront que les nouveaux pays membres perturbent, ou menacent de perturber, leur marché du travail, pourront prolonger la période de limitations d'encore deux ans, arrivant ainsi au total à une période légale « de transition » de sept ans.

9. En particulier pour les pays de l'espace Schengen : Allemagne, Belgique, Pays-Bas, Luxembourg, France, Espagne, Portugal, Grèce, Finlande, Autriche, Italie, Suède, Danemark.

10. Des traités bilatéraux sur les travailleurs migrants existent actuellement entre les pays candidats et la plupart des pays membres de l'Union européenne.

11. En particulier, l'Institut allemand d'économie de Berlin, avec notamment une étude parue en janvier 2001, l'Institut de recherche économique de Munich et l'Institut de recherche économique de Vienne ont été les plus actifs.

12. Un débat public fut organisé en Allemagne. Malgré les opinions divergentes, notamment la prise de position du patronat en faveur de l'ouverture (attiré par une main-d'œuvre qualifiée et bon marché), le pouvoir politique a décidé d'insister pour l'adoption d'une période de transition.

13. Nous ne citerons pas les résultats des sondages réalisés par les instituts allemands ; il est cependant à noter qu'ils ont relevé des pourcentages élevés - souvent supérieurs 
à $50 \%$ des personnes interrogées - de travailleurs « ayant l'intention » d'émigrer en Allemagne.

14. Le plus grand crédit est généralement accordé à celles de l'OCDE et de l'UE.

15. Le Deutsche Institut für Wirtschaft estime le nombre total des travailleurs immigrés de l'Est dans l'UE après l'autorisation de la libre circulation à 350000 par an.

16. L'Espagne et le Portugal ont rejoint la Communauté européenne en 1986. Leurs ressortissants ont alors été soumis à une période de transition de sept ans avant de pouvoir accéder en tant que travailleurs européens au marché du travail dans les autres États membres. La Grèce, qui a adhéré en 1981, s'est également vu imposer une telle période de transition.

17. Selon cette source, le pourcentage est le plus bas en Bulgarie $67,1 \%$, et le plus élevé en République tchèque $86,1 \%$. Pour l'UE, c'est le Royaume-Uni qui affiche le taux le plus élevé $80,7 \%$; suivent les pays scandinaves : Danemark $78 \%$, Suède $77,2 \%$, Finlande $73,2 \%$. L'Espagne et le Portugal se trouvent en bas du classement, avec respectivement $37,1 \%$ et $21,6 \%$. Les données concernent la population (hommes et femmes) de 25 à 64 ans en 2000 .

18. Le même constat se trouve dans une étude de l'OCDE, International Mobility of the Highly Skilled, OECD, Paris, 2003.

19. Nous reprenons la formule de Jean-Marc Ferry (Ferry et Thibaud, $1992: 213$ ).

\section{RÉSUMÉS}

L'Union européenne, sous l'impulsion de l'Allemagne et de l'Autriche, a décidé de ne pas ouvrir son marché du travail aux ressortissants des futurs pays adhérents de l'Est. Fondé sur des enquêtes portant sur l'élargissement de l'Union européenne, menées notamment en l'Estonie, en Pologne et en Hongrie auprès des milieux politico-administratifs et des experts, l'article tente de mettre en lumière la construction de représentations et de catégories liées à cette problématique. Ces dernières ressortissent à l'un ou à l'autre des deux grands registres, celui des normes construites comme objectives et celui des subjectivités. L'analyse du traitement de la question de la libre circulation des travailleurs de l'Est au sein de l'Union européenne permet de dresser un tableau de la structuration des représentations et des valeurs en jeu, ainsi que de rattacher cet exemple à un mode général d'appréhender des populations perçues comme différentes. Quelles comparaisons avec d'autres populations immigrées seraient-elles pertinentes et dans quelle mesure? En quoi les décalages consistent-ils et comment sont-ils abordés par l'expertise et les décideurs politiques ? Pourquoi, au bout du compte, le message objectiviste de l'expertise se trouve-t-il submergé par son propre hors-texte?

European Fears, Malthusian Fears. Following Germany and Austria positions, the European Union decided against opening its labour market to the nationals of the newly admitted Eastern European countries. The article, based on studies over the EU enlargement inside the politics, government, and experts circles in Estonia, Poland, and Hungary, tries to clarify the construction of representations and categories related to the question of opening the European labour market. These representations and categories belong to two large groups, either as norms - built as objective - or as subjectivities. The analysis of the way the Eastern Europe workers free 
movement inside the $\mathrm{EU}$ is treated gives us a comprehensive view on the structuring of representations and values at stake. It also enables us to link this example to a general mode of thinking of populations perceived as different. Which comparisons to other immigrant populations would be relevant, to which extent? What are the differences between the Eastern European populations and some former European immigrants, and how are they tackled by experts and politicians? Why is the experts' objectivist message eventually swamped with its own out-of-text content?

Miedos europeos, miedos maltusianos. Bajo la impulsión de Alemania y de Austria, la Unión Europea ha tomado la decisión de no abrir su mercado de trabajo a las personas originarias de los futuros Estados miembros de Europa del Este. Sobre la base de encuestas centradas en la adhesión y dirigidas a círculos políticos y administrativos así como a expertos de Estonia, Polonia y Hungría, este artículo trata de elucidar la construcción de representaciones y de categorías ligadas a la apertura del mercado europeo del trabajo. Estas categorías pueden ser clasificadas en dos grandes registros, el registro de las normas construidas objetivamente y el registro de la subjetividad. El análisis del tratamiento de la cuestión de la libre circulación de los trabajadores del Este permite, en primer lugar, elaborar un cuadro de la estructuración de las representaciones y de los valores en juego. En segundo lugar, este análisis permite ligar el ejemplo seleccionado con un modelo general de comprensión de poblaciones percibidas como diferentes. ¿Qué tipo de comparaciones con otras poblaciones inmigrantes resultaría pertinente y en qué medida? ¿En qué consisten los desfases y de qué manera son abordados por los expertos y por los responsables políticos? ¿Por qué, a fin de cuentas, el mensaje objetivo del experto se encuentra sumergido en su propio fuera de texto?

\section{AUTEUR}

\section{DETELINA TOCHEVA}

Doctorante en anthropologie sociale et ethnologie à l'EHESS, membre du LAIOS, EHESS/MSH, 54 boul. Raspail 75006 Paris. 